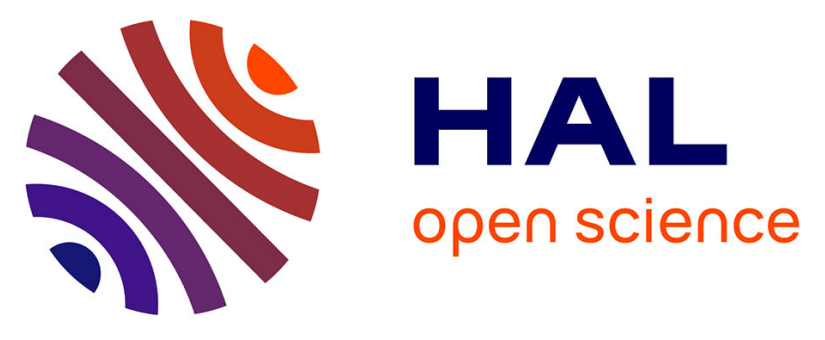

\title{
Effect of recovery mode on postexercise vagal reactivation in elite synchronized swimmers
}

Karine Schaal, Yann Le Meur, François Bieuzen, Odile Petit, Philippe

Hellard, Jean-François Toussaint, Christophe Hausswirth

\section{- To cite this version:}

Karine Schaal, Yann Le Meur, François Bieuzen, Odile Petit, Philippe Hellard, et al.. Effect of recovery mode on postexercise vagal reactivation in elite synchronized swimmers. Applied Physiology, Nutrition, and Metabolism, 2013, 38 (2), pp.126 - 133. 10.1139/apnm-2012-0155 . hal-01775076

\section{HAL Id: hal-01775076 https: / hal-insep.archives-ouvertes.fr/hal-01775076}

Submitted on 27 Apr 2018

HAL is a multi-disciplinary open access archive for the deposit and dissemination of scientific research documents, whether they are published or not. The documents may come from teaching and research institutions in France or abroad, or from public or private research centers.
L'archive ouverte pluridisciplinaire HAL, est destinée au dépôt et à la diffusion de documents scientifiques de niveau recherche, publiés ou non, émanant des établissements d'enseignement et de recherche français ou étrangers, des laboratoires publics ou privés. 


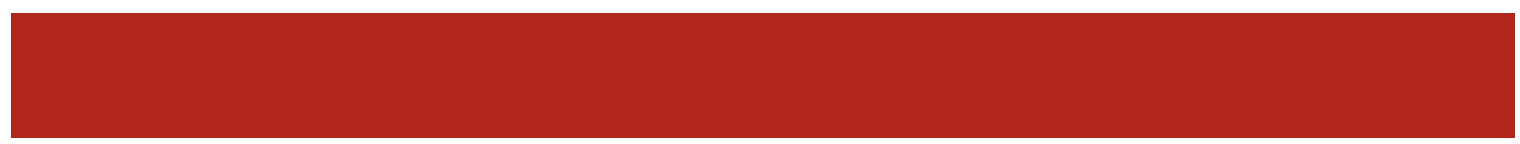

\title{
Effect of recovery mode on postexercise vagal reactivation in elite synchronized swimmers
}

Karine Schaal, Yann Le Meur, François Bieuzen, Odile Petit, Philippe Hellard, Jean-François Toussaint, and Christophe Hausswirth

\begin{abstract}
This study investigated the effect of whole-body cryostimulation (WBC), contrast-water therapy (CWT), active recovery (ACT), and passive condition (PAS) protocols on the parasympathetic reactivation and metabolic parameters of recovery in elite synchronized swimmers who performed 2 simulated competition ballets (B1 and B2) separated by 70 min. After determining maximal oxygen consumption $\left(\dot{V}_{2 \max 400}\right)$ and blood lactate concentrations $\left(\left[\mathrm{La}^{-}\right]_{\mathrm{b} 400}\right)$ during a $400-\mathrm{m}$ swim trial, 11 swimmers performed 1 protocol per week in randomized order. Heart rate variability (HRV) was measured at rest (PreB1), 5 min after B1 (PostB1), before B2 (PreB2), and 5 min after B2 (PostB2). $\mathrm{VO}_{2 \text { peak }}$ was measured at PostB1 and PostB2, and [ $\left.\mathrm{La}^{-}\right]_{\mathrm{b}}$ was measured at PostB1, PreB2, and PostB2. PostB1 $\dot{V} \mathrm{O}_{2 \text { peak }}$ and $\dot{V} \mathrm{O}_{2 \max 400}$ were similar, but PostB1 $\left[\mathrm{La}^{-}\right]_{\mathrm{b}}$ was higher than $\left[\mathrm{La}^{-}\right]_{\mathrm{b} 400}(p=0.004)$. Each ballet caused significant decreases in HRV indices. At PreB2, all HRV indices had returned to PreB1 levels in the CWT, PAS, and ACT protocols, whereas the WBC protocol yielded a 2- to 4-fold increase in vagal-related HRV indices, compared with PreB1. WBC and ACT both increased [ $\left.\mathrm{La}^{-}\right]_{\mathrm{b}}$ recovery, compared with PAS ( $p=0.06$ and $p=0.04$, respectively), and yielded an increased $\dot{V} \mathrm{O}_{\text {2peak }}$ from B1 to B2; however, it decreased after PAS $(+5.4 \%,+3.4 \%$, and $-3.6 \% ; p<0.01)$. This study describes the physiological response to repeated maximal work bouts that are highly specific to elite synchronized swimming. In the context of short-term recovery, WBC yields a strong parasympathetic reactivation, and shows similar effectiveness to ACT on the metabolic parameters of recovery and subsequent exercise capacity.
\end{abstract}

Key words: whole-body cryotherapy, female athlete, heart rate variability, maximal exercise, water immersion, active recovery.

Résumé : Cette étude analyse l'effet de la cryostimulation de l'organisme en entier ( $W B C »)$, de la thérapie par contraste de température ( $\mathrm{CWT} »)$, des protocoles actifs (ACT) et passifs (PAS) sur la réactivation sympathique et les paramètres métaboliques de la récupération chez des nageuses synchronisées de niveau élite réalisant deux chorégraphies (B1 et B2) en compétition simulée à 70 min d'intervalle. Après l'évaluation de la consommation maximale d'oxygène $\left(\dot{V}_{2 \text { max400 }}\right)$ et de la concentration sanguine de lactate ( $\left.\left[\mathrm{La}^{-}\right]_{\mathrm{b} 400}\right)$ au cours d'une épreuve de $400 \mathrm{~m}$ à la nage, 11 nageuses participent de façon aléatoire à toutes les conditions expérimentales à raison d'une par semaine. On évalue la variabilité du rythme cardiaque (HRV) au repos (PreB1), 5 min après B1 (PostB1), avant B2 (PreB2) et 5 min après $\mathrm{B} 2$ (PostB2). On évalue le $\dot{V O}_{2}$ de pointe à PostB1 et à PostB2 de même que la [La']b à PostB1, PreB2 et PostB2. À PostB1, le $\dot{V} \mathrm{O}_{2}$ de pointe est similaire au $\mathrm{VO}_{2 \max 400}$, mais la $\left[\mathrm{La}^{-}\right] \mathrm{b}$ est supérieure à $\left[\mathrm{La}^{-}\right]_{\mathrm{b} 400}(p=0,004)$. Chaque chorégraphie suscite une diminution significative des indices de HRV. À PreB2 dans les conditions CWT, PAS et ACT, tous les indices de HRV présentent des valeurs observées à PreB1, mais dans la condition WBC, on observe 2 à 4 fois plus d'augmentation de la valeur des indices HRV associés aux fonctions vagales, comparativement à PreB1. Dans les conditions WBC et ACT, on observe une augmentation de la [La- $]_{b}$ de récupération comparativement à la condition PAS $(p=0,06$ et $p=0,04)$; de plus, dans les conditions WBC et ACT, on observe une augmentation du $\dot{V} \mathrm{O}_{2}$ de pointe de $\mathrm{B} 1$ à $\mathrm{B} 2$, lequel diminue après PAS $(+5,4 \%,+3,4 \%$ et $-3,6 \%, p<0,01)$. Cette étude décrit des ajustements physiologiques à des séances répétées d'exercice maximal spécifique à la natation synchronisée de niveau élite. Dans un contexte de récupération brève, WBC révèle une importante réactivation parasympathique et s'avère aussi efficace qu'ACT dans la récupération des variables métaboliques et de la capacité physique subséquente. [Traduit par la Rédaction]

Mots-clés : cryothérapie globale, athlète féminine, variabilité du rythme cardiaque, exercice maximal, immersion dans l'eau, récupération active.

\section{Introduction}

Synchronized swimming is an aesthetic and highly technical judged sport that has become increasingly physically demanding over the past decades. In addition to developing artistic, technical, and acrobatic skills, elite synchronized swimmers must follow a high-volume, high-intensity regimen to optimize strength, flexibility, and aerobic and anaerobic exercise capacity (Liang et al. 2005; Mountjoy 2009) because these physiological parameters are directly correlated to performance scores in elite competition settings (Yamamura et al. 1999). At the international level, synchronized swimmers usually perform 2 or more training sessions per day, with few days of rest (Liang et al. 2005). Hence, in the practical scheduling of so many training hours, the time spans allotted for physical recovery between training sessions can be fairly short. Likewise, during competitions, the various ballet performances are sometimes closely scheduled, and accelerating the postexercise return to resting physiological levels after each performance is particularly important to maintain a consistent level of execution throughout the competition.

K. Schaal. Research Department, National Institute of Sport, Expertise and Performance (INSEP), Paris, France; Sports Biomedical and Epidemiological Research Institute (IRMES), National Institute of Sport, Expertise and Performance (INSEP), Paris, France.

Y. Le Meur, F. Bieuzen, and C. Hausswirth. Research Department, National Institute of Sport, Expertise and Performance (INSEP), Paris, France. O. Petit and P. Hellard. French Swimming Federation (FFN), Pantin, France.

J.-F. Toussaint. Sports Biomedical and Epidemiological Research Institute (IRMES), National Institute of Sport, Expertise and Performance (INSEP), Paris, France;

Paris-Descartes University, School of Medicine, Paris, France; Sports Medicine Investigation Center (CIMS), Hôtel-Dieu, Assistance Publique des Hôpitaux de Paris, Paris,

Paris-Desce.

Corresponding author: Christophe Hausswirth (e-mail: Christophe.Hausswirth@insep.fr). 
Heart rate variability (HRV) analysis is an established method used to gauge the extent of autonomic recovery from intense exercise sessions (Pichot et al. 2000, 2002). During exercise, the intensity-dependent withdrawal of parasympathetic tone and the increase in sympathetic activity results in an increase in heart rate and a decrease in vagal-related HRV indices (Cottin et al. 2004). After completing a given exercise bout, a rapid decrease in sympathetic activity and the return of parasympathetic heart activity to resting levels suggests a relative systemic recovery from the physiological stress imposed by the workload (Seiler and Kjerland 2006; Seiler et al. 2007). The amount of time necessary for full parasympathetic reactivation after exercise can be significantly influenced by several factors, including exercise intensity (Seiler et al. 2007; Stuckey et al. 2012) and cardiorespiratory fitness (Seiler et al. 2007; Sandercock et al. 2005). Acute high-intensity exercise and longer periods of intensified training have been shown to disrupt HRV indices at rest; in turn, these disruptions have been associated with increased fatigue, decreased sleep quality, and decreased performance (Al Haddad et al. 2009; Hynynen et al. 2006; Hynynen 2010; Garet et al. 2004; Plews et al. 2012 Uusitalo et al. 2000). A number of studies have demonstrated that different recovery techniques used in sports training, such as cold, contrasted, or thermoneutral water immersion, significantly aid parasympathetic reactivation after intense exercise in welltrained athletes (Buchheit et al. 2009a; Al Haddad et al. 2010a; Stanley et al. 2011). Cold exposure and water immersion have both been found to suppress cardiac sympathetic activity and augment parasympathetic modulation as a result of arterial baroreflex activation (Pump et al. 2001). Cold stimulation triggers peripheral vasoconstriction, leading to a shift in blood volume toward the core (Shibahara et al.1996). This increased central volume leads to increased cardiac output, stroke volume, and arterial pressure. This activates the arterial high-pressure and cardiopulmonary low-pressure baroreceptors, which are responsible for reducing sympathetic nerve activity while shifting autonomic heart rate control toward a parasympathetic dominance (Pump et al. 2001). During water immersion, regardless of temperature, the hydrostatic pressure exerted on the body provides a mechanical stimulus, which contributes to the central shift of blood volume and, hence, results in baroreflex activation (Stanley et al. 2011; Al Haddad et al. 2010a).

Although the effect of cold exposure on postexercise parasympathetic reactivation is usually studied using cold-water immersion protocols, the effect of dry-air whole-body cryostimulation $\left(\mathrm{WBC} ;-110^{\circ} \mathrm{C}\right.$ ) on postexercise autonomic recovery has not been documented, even though this recovery method is increasingly used in high-level sports (Hausswirth et al. 2011; Pournot et al. 2011). Further, the influence of WBC on parameters of metabolic recovery and subsequent maximal exercise capacity has not been evaluated.

In light of the necessity to optimize short-term recovery in a sport as seldom studied as synchronized swimming, we aimed to describe the autonomic and metabolic responses of elite synchronized swimmers to 4 different recovery methods used in sports training between 2 closely scheduled competition ballets: dry-air WBC, contrast-water therapy (CWT), active recovery (ACT), and a passive (control) condition (PAS). We compared the effect of these 4 protocols on postexercise parasympathetic reactivation, on metabolic and subjective parameters of recovery, and on subsequent maximal aerobic and anaerobic work capacity. We hypothesized that WBC would yield a significantly larger increase in vagalrelated $\mathrm{HRV}$ indices than all other recovery modes, and that WBC, ACT, and CWT would all be associated with favorable changes in metabolic parameters of recovery, compared with PAS.

\section{Materials and methods}

\section{Subjects}

Eleven highly trained elite female athletes, composing the French synchronized swimming team, took part in the study 6 months before the 2011 World Swimming Championships in Shanghai, China. The mean age of the swimmers was $20.3 \pm 1.8$ years, mean height was $170.1 \pm 4.8 \mathrm{~cm}$, and mean body weight was $61.1 \pm 4.6 \mathrm{~kg}$. All participants were familiar with exercise testing and the site of the experiment, as all procedures took place at their usual training site. The study conformed to ethical guidelines of the Declaration of Helsinki, and the study was approved by a local human research ethics committee (Ile de France XI, France; Ref. 200978) prior to beginning the study. Every athlete on the team volunteered to participate and provided their informed consent in written form.

\section{Experimental design}

The testing period began after 10 consecutive days of recovery, following a 2-week training retreat, so that subjects were fit and well rested. After an initial 400-m freestyle swimming time trial to evaluate maximal oxygen consumption $\left(\dot{V}_{2}{ }_{2 \max 400}\right)$ and its related swim velocity $\left(\dot{V} \mathrm{O}_{2 \max 400}\right.$ ) (Lavoie and Montpetit 1986; Carré et al. 1994), each subject completed 4 test sessions, spread evenly over 5 weeks, during the precompetition period (end of January to early March). Each test session exposed the swimmers to a different recovery method between 2 identical maximal exercise bouts (their technical competition ballet, lasting approximately $3 \mathrm{~min}$ ), performed 70 min apart. This recovery duration was chosen to mimic the time constraints that can occur during a competition for athletes taking part in multiple events (duo, solo, team, combination), and was the minimum duration that allowed us to perform all of the necessary tests and recovery interventions between the 2 ballets. All swimmers were tested on the same days each week, following a complete day of rest, and at the same time of day. The order in which each subject underwent the 4 different recovery protocols was randomized to reduce any possible effect of improved fitness or habituation to the protocol over these 5 weeks, and the number of subjects being tested with each recovery method was uniform throughout the experimentation period. Because all swimmers participating in the study were on a regular oral contraceptive treatment, we did not take menstrual cycle phases into consideration when planning the test sessions. Subjects were only allowed to consume water ad libitum during the test sessions. The volume of water consumed was calculated by weighing the water bottles with a food scale, and the subjects' weight fluctuations from the beginning to the end of each session were recorded.

\section{Exercise testing}

Figure 1 outlines the procedures followed during each test session. After their usual precompetition warm-up ( $45 \mathrm{~min}$ ) and mental preparation (5 $\mathrm{min}$ ), each subject performed their technical ballet routine individually (B1, 3 min in duration, with phases of apnea adding up to $64 \%$ of the total time). The second ballet (B2, identical routine) was preceded by a shorter warm-up period (15 min). At the end of each ballet, after performing the final underwater figure, the subjects remained under water as they swam to the edge of the pool, where they started breathing directly into the mask of the Cosmed $\mathrm{K} 4 \mathrm{~b}^{2}$ portable telemetry system (Rome, Italy); this method has been previously validated (Hausswirth et al. 1997). Ventilatory and gas-exchange variables were collected over 4 breathing cycles, and peak oxygen consumption $\left(\dot{V} \mathrm{O}_{2 \text { peak }}\right)$ was calculated using backward extrapolation of the $\mathrm{O}_{2}$ recovery curve (Lavoie and Montpetit 1986; Carré et al. 1994; Montpetit et al. 1981). Immediately before and after each ballet, subjects provided their global rating of perceived exertion $\left(\mathrm{RPE}_{\mathrm{g}}\right)$ and muscle aches $\left(\mathrm{RPE}_{\mathrm{m}}\right)$ on a 6- to 20-point Borg 
Fig. 1. Schema of the testing protocol followed during each session, with the order of events listed from top to bottom, then left to right. B1, B2, simulated competition ballets; ACT, active recovery; CWT, contrast-water therapy; HRV, heart rate variability; La', lactate concentration; $\mathrm{PAS}$, passive condition; $\mathrm{RPE}_{\mathrm{g}}$, global rating of perceived exertion; $\mathrm{RPE}_{\mathrm{m}}$, rating of perceived exertion for muscle aches; $\dot{V O}_{2}$, oxygen consumption; WBC, whole-body cryostimulation.

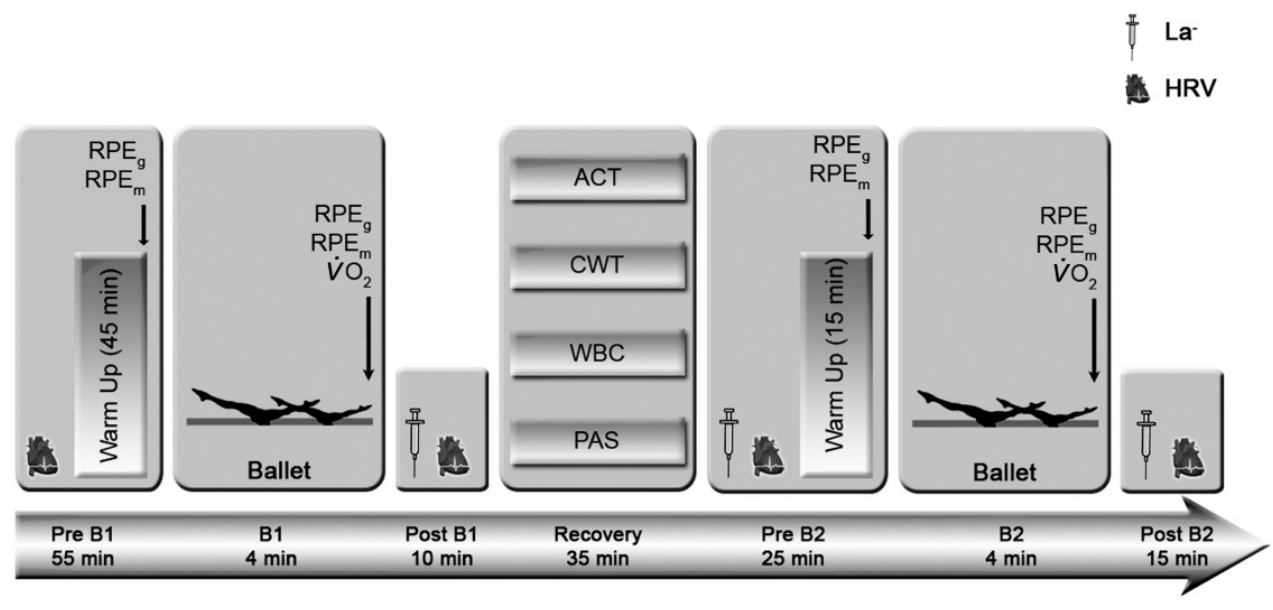

scale (Borg 1970). To encourage maximal effort during each ballet, the coaches scored performance using 4 different 20-point scales commonly used in their practices to assess the swimmers' level of performance: precision, height, energy-displacement, and homogeneity.

\section{Recovery protocols}

Subjects performed 1 of the following 4 recovery methods during each testing session.

\section{Passive condition}

For the PAS protocol, subjects were instructed to lie quietly in a lounge chair placed near the pool for $30 \mathrm{~min}$.

\section{Contrast-water therapy}

For the CWT protocol, subjects walked to the hydrotherapy unit, located in the same building as the pool, where they performed alternating cycles of cold and warm water immersion: 1 min standing in a cold-water bath $\left(9^{\circ} \mathrm{C}\right)$ and 1 min sitting in a warm-water bath $\left(39^{\circ} \mathrm{C}\right)$, immersed to the midsternal level for both, and beginning and ending with cold immersion (8 cold immersions and 7 warm immersions).

\section{Active recovery}

For the ACT protocol, 15 min of freestyle swimming at a speed corresponding to $40 \%$ of $\dot{V} \mathrm{O}_{2 \max }$ (feedback on speed was given every $50 \mathrm{~m}$ by a coach) was followed by $15 \mathrm{~min}$ of light-intensity technical synchronized swimming exercises in the water $\left(R P E_{g}\right.$ remaining below 13 on the Borg scale (Borg 1970; Toubekis et al. 2008).

\section{Whole-body cryostimulation}

For the WBC protocol, sessions were administered onsite in a specially built WBC unit (Zimmer MedizinSysteme GmbH, Ulm, Germany), consisting of 3 consecutive temperature-controlled chambers $\left(-10,-60\right.$, and $\left.-110^{\circ} \mathrm{C}\right)$. Preliminary medical clearance was obtained to ensure that none of the subjects had any contraindications to performing the WBC protocol. All swimmers performed at least 1 session of WBC before the study began, and were therefore familiar with the procedure. After all PostB1 measurements were obtained, subjects thoroughly dried their body and hair and dressed warmly before being taken by car $(>800 \mathrm{~m})$ to the WBC unit; this was done to eliminate the walk from the pool in cold weather. They then changed into a dry swimsuit, were fitted with a mask covering the nose and mouth to protect the airways, and donned an ear band, gloves, socks, and slippers to protect the extremities. Subjects passed through the 2 warmer rooms to reach the therapy room, where they remained for exactly $3 \mathrm{~min}$, walking and moving their arms slowly while being supervised by a technician.

At the end of each recovery period, just before B2, subjects marked, on a $10-\mathrm{cm}$ visual analog scale, their perception of the recovery period's effectiveness, relative to their readiness to perform the second ballet ( 0 , completely ineffective; 10 , extremely effective).

\section{HRV analysis}

Subjects were fitted with heart rate monitors (Suunto Oy, Vantaa, Finland), and heart rate was collected in R-R interval mode for 6 min while the subject sat quietly in an isolated room at 4 distinct time points for each protocol: upon arrival before beginning their initial warm-up (PreB1); 6 min after the end of B1 (PostB1); 55 min after the end of B1, following the recovery protocol (PreB2); and $6 \mathrm{~min}$ after the end of B2 (Post B2). Data from the last $256 \mathrm{~s}$ of each sampling period were used for analysis, to allow the heart rate to stabilize once subjects assumed the seated position. To avoid influencing heart rate recovery after exercise, no particular breathing frequency was imposed (Buchheit et al. 2007). For all HRV samples, it was subsequently verified that the respiration rate was always in the high-frequency $(\mathrm{HF})$ range $(>0.15-0.50 \mathrm{~Hz})$.

Data were transferred to a computer, using the Suunto Training Manager software, and were analyzed using specialized HRV analysis software (Nevrokard aHRV, Izola, Slovenia). Data were visually inspected to identify artifacts and occasional ectopic beats, which were removed manually. All HRV data were processed by the same individual. The time-varying HRV indices kept for analysis were the root-mean square difference of successive normal $\mathrm{R}-\mathrm{R}$ intervals ( $\mathrm{rMSSD}$ ), and the short-term R-R interval variability index, SD1. The standard deviation around the minor axis of the Poincaré scatterplot obtained by plotting the length of the $n$th $\mathrm{R}-\mathrm{R}$ interval against the length of its preceding $(n-1)$ th R-R interval. Mean heart rate $\left(\mathrm{HR}_{\text {mean }}\right)$ was also analyzed. Power spectral density analysis was then performed using a fast Fourier transform with a nonparametric algorithm. The power density of HF (>0.15-0.50 Hz, reflecting parasympathetic activity) and lowfrequency (LF; $0.04-0.15 \mathrm{~Hz}$, reflecting mixed sympathetic and parasympathetic activity) components of the spectrum were calculated by integrating the power density within their respective frequency bands. The ratio of LF to HF, an indicator of relative sympathovagal balance, was retained for analysis. 


\section{Metabolite analysis}

Blood samples were obtained from the finger tip, using a portable device (Lactate Pro LT-1710), to measure blood lactate concentration $\left(\left[\mathrm{La}^{-}\right]_{\mathrm{b}}\right)$ (Lactate Pro, Arkray, Kyoto, Japan) at 6 time points: before B1 (PreB1); 4 min after B1 (PostB1 ${ }_{4}=$ ); before B2 (PreB2); and 4, 10, and 15 min after B2 (PostB2 ${ }_{4}=$, PostB2 ${ }_{10}=$, and PostB2 $_{15}=$, respectively). For the $400 \mathrm{~m}$ swim time trial, $\left[\mathrm{La}^{-}\right]_{\mathrm{b}}$ was measured $4 \mathrm{~min}$ after completing the swim.

\section{Statistical analysis}

Because this study involved a relatively small number of subjects and the data obtained did not always meet the assumptions of normality, as assessed visually by normal probability plot and by the Shapiro-Wilk test, nonparametric statistical analyses were conducted. To evaluate the effect of each exercise bout and recovery, within-protocol time differences in metabolic variables and HRV indices were evaluated using Friedman's ANOVA. If a time effect was significant, Wilcoxon's test was applied in a post hoc analysis to determine which specific time points differed from the others. Between-protocol comparisons were performed, using Wilcoxon's test, to determine whether there were any differences during the recovery period between the ACT, CWT, WBC, and PAS protocols. To isolate the effect of the recovery intervention, this analysis was performed on the difference between PreB2 and PostB1, represented as il “variable" ${ }_{\text {rec }}$ (i.e., $\mathrm{ilHF}_{\mathrm{rec}}$ il $\left[\mathrm{La}^{-}\right]_{\mathrm{b}} \mathrm{rec}$, ilRPE $_{\mathrm{m} \mathrm{rec}}$, etc.). Results are reported as the means \pm SD for the parameters with normal distribution (il $\mathrm{VO}_{2 \text { peak, }}$ il $\left[\mathrm{La}^{-}\right]_{\mathrm{b} \mathrm{rec}}$ ); otherwise, results are expressed as the median and the values of the lower and upper quartiles. Spearman's coefficient of rank correlation was used to determine whether there were correlations between physiological, subjective, and autonomic variables of recovery and repeated maximal exercise. All analyses were performed using Statistica (version7.1, StatSoft France). The level of significance was set at $p<0.05$ for all analyses.

\section{Results}

\section{Exercise description}

Mean completion time for the $400 \mathrm{~m}$ swimming time trial was $308 \pm 8 \mathrm{~s}$, with a mean $\dot{V} \mathrm{O}_{2 \max 400}$ of $62.1 \pm 3.0 \mathrm{~mL} \cdot \mathrm{kg}^{-1} \cdot \mathrm{min}^{-1}$. During the 5 weeks of experimentation, no changes in any baseline or exercise variables were detected according to the week of testing. There were no differences in the amount of water consumed (885 $\pm 388 \mathrm{~mL}$ ) or in the subjects' weight fluctuations $(0.3 \pm 0.4 \mathrm{~kg})$ over the course of the study.

Because physiological parameters measured at PostB1 $\left(\mathrm{V}_{\mathrm{O}_{2 \text { peak }}}\right.$, $\left[\mathrm{La}^{-}\right]_{\mathrm{b}}$ ) were similar for all protocols, values were pooled for comparison with $400 \mathrm{~m}$ time-trial values. During the ballet (182 s), $\dot{V} \mathrm{O}_{2 \text { peak }}$ was slightly but not significantly lower than $\dot{V} \mathrm{O}_{2 \max 400}$ (60.4 $\left.\pm 2.0 \mathrm{~mL} \cdot \mathrm{kg}^{-1} \cdot \mathrm{min}^{-1}, p=0.08\right)$, and $\left[\mathrm{La}^{-}\right]_{\mathrm{b}}$ was significantly higher at PostB1 ${ }_{4}=$ than at the end of the $400 \mathrm{~m}$ time trial $(11.0 \pm$ $1.9 \mathrm{mmol} \cdot \mathrm{L}^{-1}$ vs. $7.6 \pm 2.0 \mathrm{mmol} \cdot \mathrm{L}^{-1}$, respectively, $p=0.004$ ).

\section{Heart rate variability}

The impact of exercise on all HRV indices and $\mathrm{HR}_{\text {mean }}$ was similar across all protocols, and there were no significant differences in these variables at PostB1 and PostB2. rMSSD, SD1, HF, and LF all decreased significantly with exercise, whereas $\mathrm{HR}_{\text {mean }}$ increased and recovered to resting levels during the 70-min recovery period (SD1, and $\mathrm{HR}_{\text {mean }}$ are shown in Fig. 2). LF/HF was the only index that did not show clear trends with exercise. WBC was the only protocol to yield a significant increase in vagal-related HRV indices and a decrease in $\mathrm{HR}_{\text {mean }}$ at PreB2, compared with resting values at PreB1 (rMMSD, $178 \% \pm 69 \%$ of PreB1 value, $p=0.012$; SD 1, $240 \% \pm 144 \%, p=0.009$; and $\mathrm{HR}_{\text {mean }}, 91 \% \pm 14 \%, p=0.047$ ). ACT was the only protocol to result in a higher $\mathrm{HR}_{\text {mean }}$ at PreB2 than at PreB1 ( $p=0.01)$. Although some HRV indices appeared not to fully return to PreB1 levels with ACT, this difference did not reach significance (SD1, $p=0.09 ; \mathrm{HF}, p=0.11$ ). Despite these differences,
Fig. 2. Evolution of SD1 (A) and mean heart rate $\left(\mathrm{HR}_{\text {mean }}\right)(\mathrm{B})$ for each ballet and recovery period. Values are means \pm SE. Box plots represent median interquartile range (IQR, Q25-Q75), and error bars are maximal and minimal observations within $1.5 \times \mathrm{IQR}$. Squares represent maximum and minimum observations above or below $1.5 \times$ IQR. *, Significantly different from PreB1 $(p<0.05)$; $\dagger$, significantly different from PAS $(p<0.05)$. In all conditions, PostB1 and PostB2 values were different from PreB1 and PreB2 values, so symbols were omitted for clarity. From light grey to black: PAS, CWT, WBC, ACT. B1, B2, simulated competition ballets; ACT, active recovery; CWT, contrast-water therapy; PAS, passive condition; SD1, short-term variability of successive R-R intervals; WBC, whole-body cryostimulation.

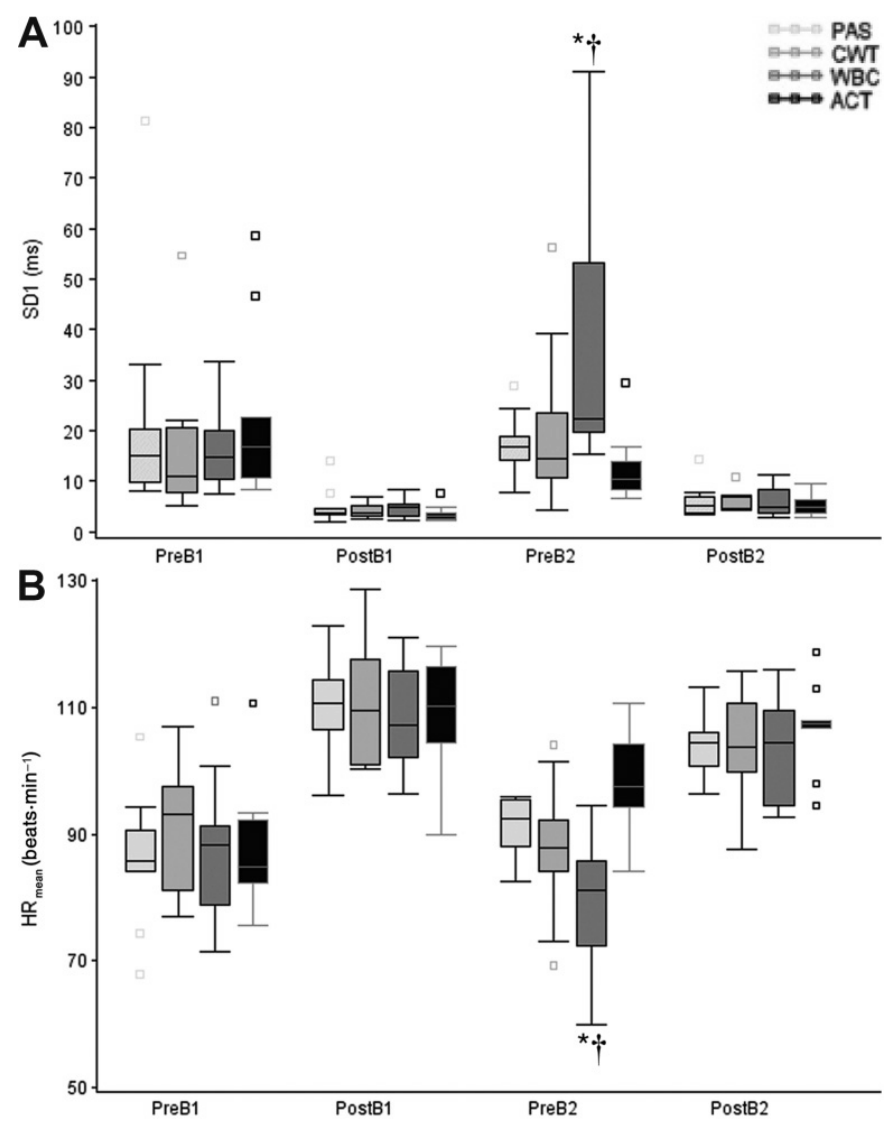

all HRV indices and $\mathrm{HR}_{\text {mean }}$ values were similar at PostB2 and PostB1, and there were no differences between protocols. When the evolution of HRV indices during the recovery period were analyzed, WBC was the only protocol to yield a significantly larger change in $\mathrm{HR}_{\text {mean }}$ and all HRV indices (ilHF ${ }_{\text {rec }}$, ilLF ${ }_{\text {rec, }}$, ilLF/HF rec are displayed in Fig.3). CWT did not differ from PAS on any of the HRV indices analyzed.

\section{Metabolic parameters}

WBC and ACT were the only protocols to yield significantly higher $\dot{V} \mathrm{O}_{2 \text { peak }}$ values at $\mathrm{B} 2$ than at $\mathrm{B} 1$, with gains of $5.4 \% \pm 3.2 \%$ and $3.4 \% \pm 2.9 \%$, respectively (Fig. 4). Every swimmer reached a higher $\dot{V} \mathrm{O}_{2 \text { peak }}$ during $\mathrm{B} 2$ after using $\mathrm{WBC}$ in recovery, and all except 1 swimmer did so with ACT. In the CWT protocol, $\dot{V} \mathrm{O}_{2 \text { peak }}$ did not differ significantly between B1 and B2. PAS was the only protocol to yield a significant decrease in $\mathrm{VO}_{2 \text { peak }}(-3.6 \% \pm 2.1 \%)$; this was observed in every subject. $\left[\mathrm{La}^{-}\right]_{\mathrm{b}}$ reached similar levels at PostB $1_{4}=$ and PostB2 $2_{4}=$, and there was no significant protocol effect (all protocols combined, $11.2 \pm 2.6 \mathrm{mmol} \cdot \mathrm{L}^{-1}$ and $10.4 \pm$ $2.1 \mathrm{mmol} \cdot \mathrm{L}^{-1}$, respectively; $\left.p=0.14\right)$. il $\left[\mathrm{La}^{-}\right]_{\mathrm{b} \text { rec }}$ was significantly larger, however, during ACT than PAS $(p=0.04)$, and nearly so for WBC than for PAS $(p=0.06)$, as shown in Fig. 5. After B2, there was 
Fig. 3. Change in power spectral density variables from PostB1 to PreB2, ilHF (A), ilLF (B), and LF/HF (C). Box plots represent median, interquartile range (IQR, Q25-Q75), and error bars are maximal and minimal observations within $1.5 \times$ IQR. Squares represent maximum and minimum observations above or below $1.5 \times$ IQR. *, Significantly different from PAS $(p<0.05)$. HF, high frequency; LF, low frequency; ACT, active recovery; CWT, contrast-water therapy; PAS, passive condition; WBC, whole-body cryostimulation.
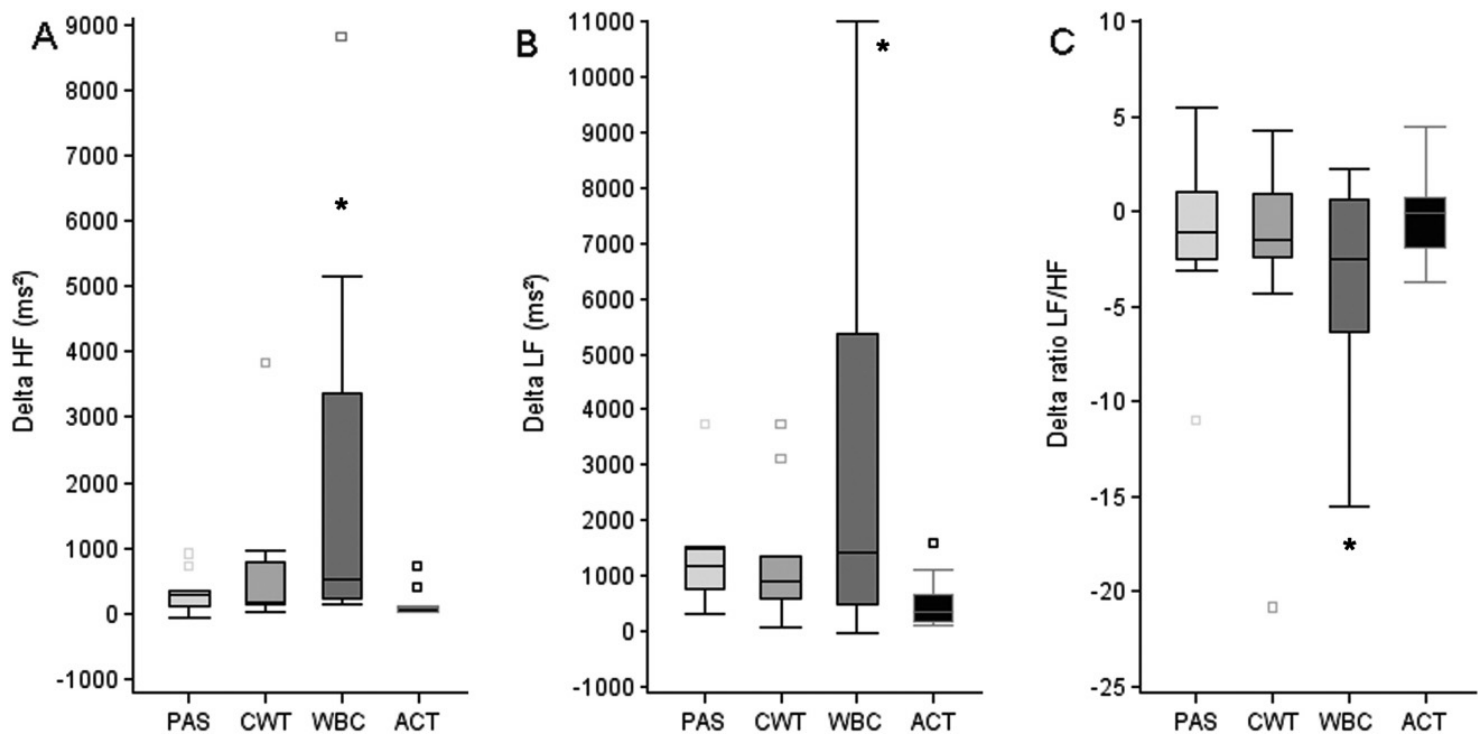

Fig. 4. il $\dot{V} \mathrm{O}_{2 \text { peak, }}$ change in peak oxygen consumption between $\mathrm{B} 2$ and B1. B1, B2, simulated competition ballets; ACT, active recovery; CWT, contrast-water therapy; PAS, passive condition; WBC, wholebody cryostimulation. *, Significantly different from baseline $(p<0.05)$; $\dagger$, significantly different from PAS $(p<0.05)$.

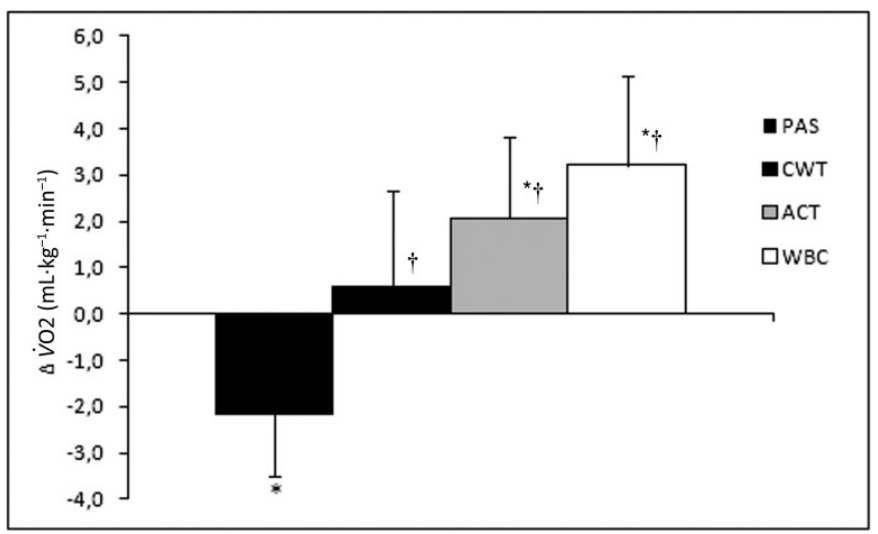

no protocol effect on the magnitude of the decrease in $\left[\mathrm{La}^{-}\right]_{\mathrm{b}}$ at PostB2 $_{10}=$ and PostB2 ${ }_{15}=$.

\section{Subjective ratings}

$\mathrm{RPE}_{\mathrm{m}}$ values peaked at PostB1 and PostB2, and returned to resting levels at the end of recovery (PreB2) for all protocols except WBC, during which $\mathrm{RPE}_{\mathrm{m}}$ remained slightly but significantly elevated, compared with PreB1 (13.4 \pm 2.0 vs. $11.9 \pm 2.5)$. PAS was the only protocol associated with significantly higher $\mathrm{RPE}_{\mathrm{m}}$ at PostB2 than at PostB1 (17.4 \pm 1.4 vs. $16.5 \pm 1.3$ ); all other protocols showed similar $\mathrm{RPE}_{\mathrm{m}}$ after each ballet. The exercise and recovery trends for $\mathrm{RPE}_{\mathrm{g}}$ were similar to those for $\mathrm{RPE}_{\mathrm{m}}$, except that $\mathrm{RPE}_{\mathrm{g}}$ values were significantly lower at PreB2 than at PreB1 after a shorter second warm-up for all protocols except WBC $(p=0.07)$. PAS was also associated with significantly lower ratings on the visual analog scale (lower perceived effectiveness of recovery) than all other protocols (PAS, $4.7 \pm 2.1$; CWT, $6.5 \pm 1.6$; ACT , $7.5 \pm 1.1$; WBC, $6.2 \pm$ $1.3 ; p<0.05)$.
Subjective performance scores

Mean scores obtained for B1 and B2 are shown in Table 1. Precision, energy-displacement, homogeneity, and overall scores were slightly but significantly lower at B2 than at B1, with a mean decrease of one-tenth of 1 point on the 20 -point scale $(-0.5 \%$ change). There was no significant effect of protocol on the difference in scores between B1 and B2.

\section{Discussion}

This study is the first to describe the physiological response of highly trained elite synchronized swimmers to 2 full-length competition ballets separated by a short recovery period. To our knowledge, this is the first time that WBC was investigated as a recovery technique between 2 closely scheduled maximal exercise bouts. The 2 most important findings of this study are that using WBC shortly after a full-length ballet resulted in a strong parasympathetic reactivation in elite swimmers, yielding 2- to 4-fold increases in vagal-related HRV indices, compared with pre-exercise values, within only $1 \mathrm{~h}$; and that WBC exerted a significant influence on the metabolic parameters of recovery and subsequent exercise, with a larger clearance of plasma lactate and an increase in maximal aerobic work output during the second ballet. The latter was only matched by the effects of active recovery.

\section{Effect of repeated exercise and recovery on HRV}

Executing an elite-level synchronized swimming ballet demands a very large physical effort, requiring maximal aerobic work production with large anaerobic contributions. Rates of aerobic energy production at the end of each ballet were similar to those obtained after the 400-m swimming time trial; however, anaerobic contribution was significantly greater at the end of each ballet than after the 400-m swimming time trial, a test considered to be an accurate evaluation of maximal aerobic capacity in swimmers (Lavoie and Montpetit 1986). Furthermore, RPE $E_{g}$ and $\mathrm{RPE}_{\mathrm{m}}$ values obtained at the end of B1 and B2 were very high, indicating that the swimmers perceived these performances to be very difficult (Borg 1970). Research on the metabolic toll of synchronized swimming ballets is scarce, especially at the elite level. Peak $\left[\mathrm{La}^{-}\right]_{\mathrm{b}}$ values (mean, $11.0 \pm 1.9 \mathrm{mmol} \cdot \mathrm{L}^{-1}$ ) obtained at the end of each ballet surpassed those reported more than a decade ago by 
Fig. 5. Mean difference in blood lactate concentration $\left[\mathrm{La}^{-}\right]_{\mathrm{b} \mathrm{rec}}$ ) for each protocol. ACT, active recovery; CWT, contrast-water therapy; PAS, passive condition; WBC, whole-body cryostimulation.

$\dagger$, Significantly different from PAS $(p<0.05)$. The difference between WBC and PAS nearly missed significance $(p=0.059)$.

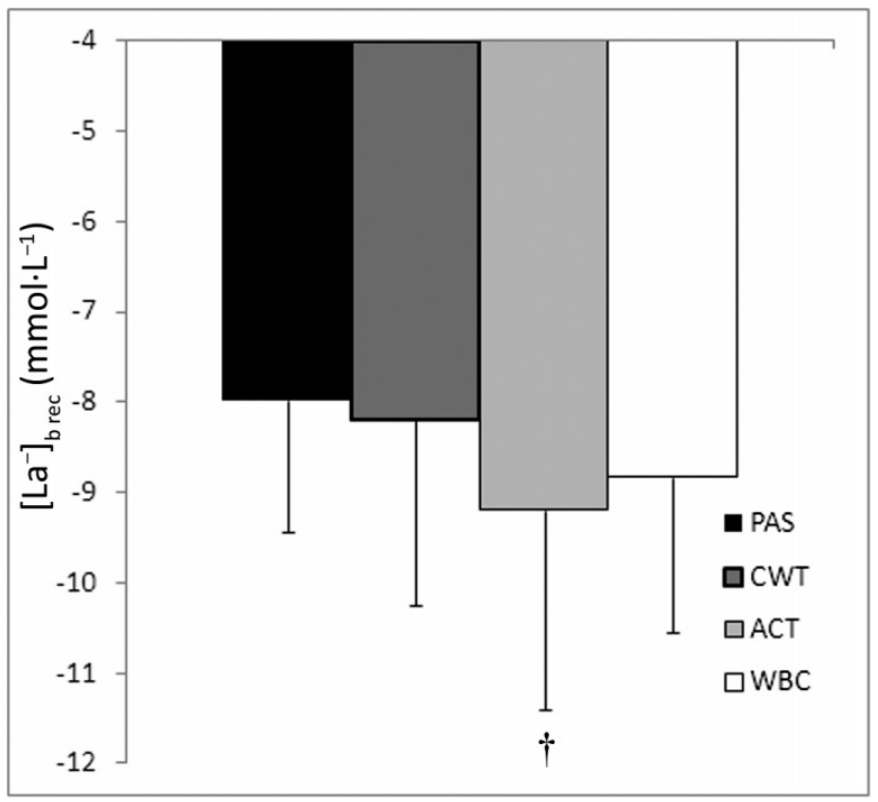

Table 1. Difference in the scores obtained between B1 and B2 for each protocol.

\begin{tabular}{|c|c|c|c|c|c|}
\hline & Height & Precision & $\begin{array}{l}\text { Energy- } \\
\text { displacement }\end{array}$ & Homogeneity & $\begin{array}{l}\text { Overall } \\
\text { score }\end{array}$ \\
\hline PAS & $0.0 \pm 0.2$ & $-0.1 \pm 0.3$ & $0.0 \pm 0.2$ & $0.0 \pm 0.4$ & $0.0 \pm 0.2$ \\
\hline ACT & $-0.1 \pm 0.3$ & $0.0 \pm 0.3$ & $0.0 \pm 0.4$ & $0.1 \pm 0.3$ & $0.0 \pm 0.3$ \\
\hline WBC & $-0.1 \pm 0.3$ & $-0.2 \pm 0.3$ & $-0.2 \pm 0.3$ & $-0.3 \pm 0.3$ & $-0.2 \pm 0.3$ \\
\hline CWT & $-0.1 \pm 0.4$ & $-0.1 \pm 0.4$ & $-0.1 \pm 0.3$ & $-0.2 \pm 0.4$ & $-0.1 \pm 0.3$ \\
\hline
\end{tabular}

Note: Overall score, mean of all 4 protocols. B1, B2, simulated competition ballets; ACT, active recovery; CWT, contrast-water therapy; PAS, passive condition; WBC, whole-body cryostimulation.

Yamamura et al. (2000), who found that $\left[\mathrm{La}^{-}\right]_{\mathrm{b}}$ in Japan National finalists only reached $4.7 \pm 1.1 \mathrm{mmol} \cdot \mathrm{L}^{-1}$, or $46 \% \pm 11 \%$ of their previously measured peak $\left[\mathrm{La}^{-}\right]_{\mathrm{b}}$. In addition, we found that the swimmers' high $\dot{V} \mathrm{O}_{2 \max }\left(62.1 \pm 3.0 \mathrm{~mL} \cdot \mathrm{kg}^{-1} \cdot \mathrm{min}^{-1}\right)$ supports their highly trained status and conveys that this sport has evolved over the years to become more physically demanding. Indeed, reported aerobic capacities of elite synchronized swimmers have evolved from $44 \pm 4 \mathrm{~mL} \cdot \mathrm{kg}^{-1} \cdot \mathrm{min}^{-1}$ in 1980 (Poole et al. 1980) to $53 \pm$ $5 \mathrm{~mL} \cdot \mathrm{kg}^{-1} \cdot \mathrm{min}^{-1}$ in 1999 (Chatard et al. 1999).

In accordance with metabolic and subjective indicators of maximal work output during the ballets, the large decrease in all vagal-related HRV indices observed from 6 to $10 \mathrm{~min}$ after the end of each ballet reflected a significant reduction in the parasympathetic modulation of heart rate after the completion of intense exercise (Cottin et al. 2004). Even though we did not measure heart rate response during exercise, previous data obtained during competitions from the French elite synchronized swimming team revealed that the swimmers reached their maximal heart rate over the course of the routine (Hausswirth 2009). In spite of the large exercise-induced shift in autonomic heart rate control, complete recovery of all vagal-related HRV indices occurred within 1hour of the end of the first ballet, attesting to the high fitness of these athletes (Seiler et al. 2007). Even though the time span required for full postexercise parasympathetic reactivation (to pre-exercise levels) is known to increase with the relative intensity of exercise (Seiler etal. 2007; Stuckey et al. 2012), greater cardiorespiratory fitness is associated with a significantly faster recovery of resting vagal tone (Seiler etal. 2007; Sandercock etal.2005).

In our swimmers, HRV indices obtained after the completion of B2 indicated that the sympathetic influence over heart rate control remaining $10 \mathrm{~min}$ after exercise was no greater than that 10 min after B1, suggesting that autonomic response to repeating the same exercise in this short time span is similar. This finding, together with the similar $\left[\mathrm{La}^{-}\right]_{\mathrm{b}}, \mathrm{RPE}_{\mathrm{g}}$, and $\mathrm{RPE}_{\mathrm{m}}$, and equal or greater $\dot{V} \mathrm{O}_{\text {2peak }}$ attained for all protocols (except $\dot{V} \mathrm{O}_{2 \text { peak }}$ after PAS), demonstrates that with WBC, ACT, and CWT, the swimmers were able to repeat the same maximal workload with similar autonomic, metabolic, and subjective responses.

\section{Effect of specific recovery techniques on HRV}

The augmentation of pre-exercise HRV values observed in synchronized swimmers after WBC reflects a strong parasympathetic reactivation at the cardiac level, with values largely surpassing those measured at rest as early as 60 min after maximal exercise. To our knowledge, this was the first study to describe such a large increase in vagal-related HRV indices from any cold exposure recovery technique used after exercise, with mean increases ranging from $78 \%$ for rMSSD and $140 \%$ for SD1 to $296 \%$ for HF. Westerlund et al. (2006) investigated the HRV response to WBC in the resting state in nonathletic women, and reported that $2 \mathrm{~min}$ of WBC $\left(-110^{\circ} \mathrm{C}\right)$ augmented HRV indices of parasympathetic activity by $53 \%$ for rMSSD and $47 \%$ for SD1. In the case of highly trained swimmers, we showed that this significant effect of WBC occurred even when the treatment was performed shortly after maximal exercise, in a context of heightened cardiac sympathetic activity and suppressed vagal tone.

Other conventional cryostimulation methods used in the context of recovery from physical training, such as shoulder-deep cold-water immersion (5 min in 11 to $14^{\circ} \mathrm{C}$ water) have been found to significantly aid postexercise parasympathetic reactivation (Al Haddad et al. 2010a; Buchheit et al. 2009a; Stanley et al. 2011) to pre-exercise levels. The additional increase in vagal modulation observed with WBC, compared with typical cold-water immersion methods, could potentially be explained by the fact that the very low temperatures in WBC imposed a larger thermal stress than immersion in $14^{\circ} \mathrm{C}$ water. However, studies quantifying relative thermal stress and changes in core temperature resulting from various modes of cryostimulation are lacking. Additionally, during WBC, the entire body is exposed to cold, including the face and neck. It has been shown that the direct effect of cold on the head alone, using face immersion in cold water (without breathholding), aids parasympathetic reactivation significantly after exercise (Al Haddad et al. 2010b). This increase in vagal tone is thought to be principally mediated by trigeminal brain stem pathways, rather than by the arterial baroreflex (Khurana and $\mathrm{Wu}$ 2006). Further, Eckberg et al. (1984) showed that stimulating trigeminal cutaneous receptors using cold-water face immersion augments the magnitude of the vagal response induced by arterial baroreflex activation alone.

CWT did not stand out as being particularly beneficial to autonomic recovery in our subjects, compared with PAS; both protocols resulted in a return to resting HRV indices. The short intermittent exposure to cold during CWT might have provided insufficient thermal stress to significantly boost parasympathetic activity beyond pre-exercise levels. Stanley et al. (2011) reported that cold-water immersion exerts a larger effect on parasympathetic reactivation than contrast-water therapy, suggesting that the more important cold stimulus augments the effectiveness of water immersion in this respect. Further, the swimmers' habituation to spending several hours each day immersed in a thermoneutral pool for their training could have reduced the impact of this recovery method in this specific population. 
The workload performed during ACT, despite its low intensity, could be expected to slow autonomic recovery after maximal exercise, because even a light exercise load would maintain a relative intensity-dependent degree of parasympathetic withdrawal and sympathetic nerve activity. However, in the case of highly aerobically trained individuals, our results demonstrate that a subsequent low-intensity active recovery does not significantly hinder parasympathetic reactivation. Five minutes after the completion of active recovery, all HRV parameters (except LF) had returned to near-baseline levels; only $\mathrm{HR}_{\text {mean }}$ remained slightly elevated. These findings remain specific to active recovery performed in water; the effect of immersion, together with the horizontal body position during swimming, probably aided parasympathetic reactivation (Buchheit et al. 2009a, 2010). In this respect, the effects of the active recovery used here may not be comparable to active recovery protocols performed on land.

Finally, during PAS, complete recovery to pre-exercise HRV indices occurred at least twice as fast as that observed by Stanley etal. (2011), who showed that HRV indices returned to pre-exercise levels after 130 min of passive recovery. This discrepancy could be attributed to a higher level of cardiorespiratory fitness (Seiler et al. 2007), but could also be attributed to different body positions. Our subjects adopted a supine position for $30 \mathrm{~min}$, whereas the subjects of Stanley et al. (2011) remained in a seated position for $10 \mathrm{~min}$. Buchheit et al. (2009b) demonstrated that lying supine led to a faster heart rate recovery after exercise than sitting.

\section{Parasympathetic reactivation and metabolic parameters of recovery and repeated exercise}

Despite the large systemic sympathetic response necessary to support maximal work production rates during exercise (Brooks and Mercier 1994), we showed that in these highly trained athletes, autonomic function at the cardiac level recovered fully within $70 \mathrm{~min}$, and that poor parasympathetic reactivation after maximal exercise was therefore not a limiting factor to recovery and subsequent exercise capacity. No link was found between the extent of parasympathetic reactivation and any metabolic or subjective parameters of recovery or subsequent exercise capacity. Specifically, no differences in parasympathetic reactivation were found between PAS, ACT, and CWT; however, aerobic work output decreased significantly from B1 to B2 after PAS $(-3.6 \% \pm 2.1 \%)$, was maintained with CWT $(+1.0 \% \pm 3.4 \%)$, and increased with ACT $(+3.4 \% \pm 2.9 \%)$. To our knowledge, only a few studies have investigated whether a larger postexercise parasympathetic reactivation, occurring as a result of cold-water immersion, is associated with improved performance in the short term (Buchheit et al. 2009a; Stanley et al. 2011). Even though in both studies cold-water immersion enhanced parasympathetic reactivation and yielded greater subjective ratings of recovery than passive conditions, no associations were found between HRV recovery and absolute performance measures, suggesting that, in the case of healthy highly trained individuals, this aspect of recovery may not affect subsequent exercise capacity. From a medical safety perspective, however, optimizing parasympathetic reactivation might help reduce the occurrence of dangerous arrhythmias, which tend to occur more readily in situations with a strong sympathetic background, such as immediately after intense exercise in the heat (Billman 2002).

To our knowledge, this was the first study to describe the effect of WBC as a short-term (i.e., $1 \mathrm{~h}$ ) recovery aid. It showed that elite athletes respond favorably to this technique in the context of repeated maximal aerobic exercise. WBC yielded results nearly similar to ACT on the parameters of metabolic recovery, aiding blood lactate clearance, and enabling a greater aerobic work output during B2, compared with PAS. It is well established that, compared with passive conditions, active recovery increases the clearance of lactate and other metabolic byproducts, such as hydrogen ions, ammonium ions, and inorganic phosphate (Banfi et al. 2010; Fairchild et al. 2003). An increased blood lactate clearance has also been reported after cold exposure, compared with passive recovery (Heyman et al. 2009). Because the removal of these ions from the bloodstream is associated with a greater ability to repeat maximal aerobic and anaerobic performance in the short term (Neric et al. 2009), our findings support the reduced effectiveness of passive recovery in this regard, confirming active recovery and cold exposure to be more appropriate options between 2 synchronized swimming performances.

In spite of the desirable effects of WBC, ACT, and CWT on the metabolic aspects of recovery and subsequent exercise capacity, these benefits were not accompanied by differences in performance scores attributed by the coaches. Even though the technical and aesthetic merit of a given ballet performance does not solely depend on maximal exercise capacity (they also depend on several aesthetic and technical characteristics), an increase in aerobic work output during the second ballet could have favorably impacted the scores attributed by coaches (such as the increased height of compulsory figures, the amplitude of movement, and the overall energy level and homogeneity of the performance). In this respect, Yamamura et al. (1999) found a significant correlation between performance scores and the physiological attributes of synchronized swimmers, including aerobic and anaerobic work capacity and the amplitude of leg movements. Thus, in spite of the sensitivity and reproducibility limitations inherent to subjective evaluations of performance in judged sports, (Damisch et al. 2006; Ansorge 1978; Ste-Marie and Lee 1991), the physiological evidence pointing to an optimized recovery with WBC and ACT encourage further investigations of sports events in which a $3 \%$ to $5 \%$ increase in $\dot{V} \mathrm{O}_{2 \text { peak }}$, as was seen here, is usually associated with improved absolute performance (such as during a cycling or running time trial (Saunders et al. 2010)).

\section{Conclusion}

This study described the autonomic and metabolic responses of elite synchronized swimmers to 2 full-length competition ballets, and their adaptations to 4 different protocols performed during the recovery period separating them. We brought forth 2 novel findings, supporting the effectiveness of WBC in the context of postexercise autonomic and metabolic recovery and repeated exercise performance. First, we demonstrated that a single session of WBC performed shortly after a maximal exercise exerted a strong influence on parasympathetic reactivation in the context of a heightened sympathetic background. Second, our results showed that, similar to ACT, WBC is associated with improved metabolic recovery after maximal exercise. Future research should try to determine whether the regular use of WBC as a recovery technique confers additional benefits over longer periods of time, because fatigue accumulation during phases of intensified training has been associated with changes in the autonomic modulation of heart rate in athletes (Pichot et al. 2002).

\section{Acknowledgements}

The authors of this study thank the French synchronized swimming athletes for participating in this study, as well as their respective coaches - Charlotte Massardier, Pascale Meyet, Anne Capron, and Julie Fabre - for their valuable assistance in scheduling this protocol within the athletes' training schedules. They would also like to thank Dr. Philippe Le Van, Jean-Robert Filliard, Marielle Volondat, and Philippe Van de Cauter for supervising the cryostimulation sessions and, finally, INSEP and the French National Swimming Federation for their support in this study.

\section{References}

Al Haddad, H., Laursen, P.B., Ahmaidi, S., and Buchheit, M. 2009. Nocturnal heart rate variability following supramaximal intermittent exercise. Int. J. Sports Physiol. Perf. 4(4): 435-447. PMID:20029095.

Al Haddad, H., Laursen, P.B., Chollet, D., Lemaitre, F., Ahmaidi, S., and Buchheit, M. 2010a. Effect of cold or thermoneutral water immersion on post-exercise heart rate recovery and heart rate variability indices. Auton. Neurosci. 156(1-2): 111-116. doi:10.1016/j.autneu.2010.03.017. PMID:20403733. Al Haddad, H., Laursen, P.B., Ahmaidi, S., and Buchheit, M. $2010 \mathrm{~b}$. Influence of 
cold water face immersion on post-exercise parasympathetic reactivation. Eur. J. Appl. Physiol. 108(3): 599-606. doi:10.1007/s00421-009-1253-9. PMID: 19882167.

Ansorge, C.J. 1978. Bias in judging women's gymnastics induced by expectations of within-team order. Res. Q.49(4): 399-405. PMID:741076.

Banfi, G., Lombardi, G., Colombini, A., and Melegati, G. 2010. Whole-body cryotherapy in athletes. Sports Med. 40(6): 509-517. doi:10.2165/11531940-00000000000000.PMID:20524715.

Billman, G.E. 2002. Aerobic exercise conditioning: a nonpharmacological antiarrhythmic intervention. J. Appl. Physiol. 92(2): 446-454. PMID:11796650.

Borg, G., 1970. Perceived exertion as an indicator of somatic stress. Scand. J. Rehab. Med. 2(2): 92-98. PMID:5523831.

Brooks, G.A., and Mercier, J. 1994. Balance of carbohydrate and lipid utilization during exercise: the "crossover" concept. J. Appl. Physiol. 76(6): 2253-2261. PMID:7928844.

Buchheit, M., Laursen, P.B., and Ahmaidi, S. 2007. Parasympathetic reactivation after repeated sprint exercise. Am. J. Physiol. Heart Circ. Physiol. 293(1): H133-H141. doi:10.1152/ajpheart.00062.2007. PMID:17337589.

Buchheit, M., Peiffer, J.J., Abbiss, C.R., and Laursen, P.B. 2009a. Effect of cold water immersion on postexercise parasympathetic reactivation. Am. J. Physiol. Heart Circ. Physiol. 296(2): H421-H427. PMID:19074671.

Buchheit, M., Al Haddad, H., Laursen, P.B., and Ahmaidi, S. 2009b. Effect of body posture on postexercise parasympathetic reactivation in men. Exp. Physiol. 94(7): 795-804. doi:10.1113/expphysiol.2009.048041. PMID:19395660.

Buchheit, M., Al Haddad, H., Chivot, A., Leprêtre, P.M., Ahmaidi, S., and Laursen, P.B. 2010. Effect of in- versus out-of-water recovery on repeated swimming sprint performance. Eur. J. Appl. Physiol. 108(2): 321-327. doi: 10.1007/s00421-009-1212-5. PMID:19795131.

Carré, F., Dassonville , J., Beillot, J., Prigent, J.-Y., and Rochcongar, P. 1994. Use of oxygen uptake recovery curve to predict peak oxygen uptake in upper body exercise. Eur. J. Appl. Physiol. Occup. Physiol. 69(3): 258-261. doi:10.1007/ BF01094798. PMID:8001539.

Chatard, J.C., Mujika, I., Chantegraille, M.C., and Kostucha, J. 1999. Performance and physiological responses to a 5-week synchronized swimming technical training programme in humans. Eur. J. Appl. Physiol. 79(6): 479-483. doi: $10.1007 / \mathrm{s} 004210050540$.

Cottin, F., Médigue, C., Leprêtre, P.M., Papelier, Y., Koralsztein, J.P., and Billat, V. 2004. Heart rate variability during exercise performed below and above ventilatory threshold. Med.Sci.Sports Exerc.36(4):594-600.doi:10.1249/01.MSS. 0000121982.14718.2A. PMID:15064586.

Damisch, L., Mussweiler, T., and Plessner, H. 2006. Olympic medals as fruits of comparison? Assimilation and contrast in sequential performance judgments. J. Exp. Psychol. Appl. 12(3): 166-178. doi:10.1037/1076-898X.12.3.166. PMID:16953743.

Eckberg, D.L., Mohanty, S.K., and Raczkowska, M. 1984. Trigeminal-baroreceptor reflex interactions modulate human cardiac vagal efferent activity. J. Physiol. 347(1): 75-83. PMID:6707976.

Fairchild, T.J., Armstrong, A.A., Rao, A., Liu, H., Lawrence, S., and Fournier, P.A. 2003. Glycogen synthesis in muscle fibers during active recovery from intense exercise. Med. Sci. Sports Exerc. 35(4): 595-602. doi:10.1249/01.MSS. 0000058436.46584.8E. PMID:12673142.

Garet, M., Tournaire, N., Roche, F., Laurent, R., Lacour, J.R., Barthélémy, J.C., et al. 2004. Individual interdependence between nocturnal ANS activity and performance in swimmers. Med. Sci. Sports Exerc. 36(12): 2112-2118. PMID: 15570148.

Hausswirth, C. 2009. In Proceedings of the 14th Annual Congress of the ECSS. 24-27 June 2009. Oslo, Norway.

Hausswirth, C., Bigard, A.X., and Le Chevalier, J.M. 1997. The Cosmed K4 telemetry system as an accurate device for oxygen uptake measurements during exercise. Int. J. Sports Med. 18(6): 449-453. doi:10.1055/s-2007-972662. PMID: 9351691.

Hausswirth, C., Louis, J., Bieuzen, F., Pournot, H., Fournier, J., Filliard, J.F., et al. 2011. Effects of whole-body cryotherapy vs. far-infrared vs. passive modalities on recovery from exercise-induced muscle damage in highly-trained runners. PLoS One, 6(12): e27749. doi:10.1371/journal.pone.0027749. PMID: 22163272.

Heyman, E., De Geus, B., Mertens, I., and Meeusen, R. 2009. Effects of four recovery methods on repeated maximal rock climbing performance. Med. Sci. Sports Exerc. 41(6): 1303-1310. doi:10.1249/MSS.0b013e318195107d. PMID: 19461534.

Hynynen, E., Uusitalo, A., Konttinen, N., and Rusko, H. 2006. Heart rate variability during night sleep and after awakening in overtrained athletes. Med. Sci. Sports Exerc. 38(2): 313-317. doi:10.1249/01.mss.0000184631.27641.b5. PMID: 16531900.

Hynynen, E., Vesterinen, V., Rusko, H., and Nummela, A. 2010. Effects of moderate and heavy endurance exercise on nocturnal HRV. Int. J. Sports Med. 31(6): 428-432. doi:10.1055/s-0030-1249625. PMID:20419617.

Khurana, R.K., and Wu, R. 2006. The cold face test: a non-baroreflex mediated test of cardiac vagal function. Clin. Auton. Res. 16(3): 202-207. doi:10.1007/ s10286-006-0332-9. PMID:16491317.

Lavoie, J.M., and Montpetit, R.R., 1986. Applied physiology of swimming. Sports Med. 3(3): 165-189. doi:10.2165/00007256-198603030-00002. PMID:3520747.
Liang, M.T., Arnaud, S.B., Steele, C.R., Hatch, P., and Moreno, A. 2005. Ulnar and tibial bending stiffness as an index of bone strength in synchronized swimmers and gymnasts. Eur.J.Appl. Physiol.94(4): 400 -407. doi:10.1007/s00421005-1351-2. PMID:15864633.

Montpetit, R.R., Léger, L.A., Lavoie, J.-M., and Cazorla, G. 1981. $\dot{V} \mathrm{O}_{2}$ peak during free swimming using the backward extrapolation of the $\mathrm{O}_{2}$ recovery curve. Eur. J Appl. Physiol. Occup. Physiol. 47(4): 385-391. doi:10.1007/BF02332966. PMID:7199441.

Mountjoy, M. 2009. Injuries and medical issues in synchronized Olympic sports. Curr. Sports Med. Rep. 8(5): 255-261. PMID:19741353.

Neric, F.B., Beam, W.C., Brown, L.E., and Wiersma, L.D. 2009. Comparison of swim recovery and muscle stimulation on lactate removal after sprint swimming. J. Strength Cond. Res. 23(9): 2560-2567. doi:10.1519/JSC. 0b013e3181bc1b7a. PMID:19910818.

Pichot, V., Roche, F., Gaspoz, J.M., Enjolras, F., Antoniadis, A., Minini, P., et al. 2000. Relation between heart rate variability and training load in middledistance runners. Med. Sci. Sports Exerc. 32(10): 1729-1736. doi:10.1097/ 00005768-200010000-00011.PMID:11039645.

Pichot, V., Busso, T., Roche, F., Garet, M., Costes, F., Duverney, D., et al. 2002. Autonomic adaptations to intensive and overload training periods: a laboratory study. Med. Sci. Sports Exerc. 34(10): 1660-1666. doi:10.1097/00005768200210000-00019. PMID:12370569.

Plews, D.J., Laursen, P.B., Kilding, A.E., and Buchheit, M. 2012. Heart rate variability in elite triathletes, is variation in variability the key to effective training? A case comparison. Eur. J. Appl. Physiol. 112: 3729-3741. doi:10.1007/ s00421-012-2354-4. PMID:22367011.

Poole, G.W., Crepin, B.J., and Sevigny, M. 1980. Physiological characteristics of elite synchronized swimmers. Can. J. Appl. Sports Sci. 5(3): 156-160. PMID: 7449027.

Pournot, H., Bieuzen, F., Louis, J., Mounier, R., Fillard, J.-R., Barbiche, E., et al. 2011. Time-course of changes in inflammatory response after whole-body cryotherapy multi exposures following severe exercise. PloS One, 6(7): e22748. doi:10.1371/journal.pone.0022748. PMID:21829501.

Pump, B., Shiraishi, M., Gabrielsen, A., Bie, P., Christensen, N.J., and Norsk, P. 2001. Cardiovascular effects of static carotid baroreceptor stimulation during water immersion in humans. Am. J. Physiol. Heart Circul. Physiol. 280(6): H2607-H2615. PMID:11356616.

Sandercock, G.R.H., Bromley, P.D., and Brodie, D.A. 2005. Effects of exercise on heart rate variability: inferences from meta-analysis. Med. Sci. Sports Exerc. 37(3): 433-439. doi:10.1249/01.MSS.0000155388.39002.9D. PMID:15741842.

Saunders, P.U., Cox, A.J., Hopkins, W.G., and Pyne, D.B. 2010. Physiological measures tracking seasonal changes in peak running speed. Int. J. Sports Physiol. Perform. 5(2): 230-238. PMID:20625195.

Seiler, K.S., and Kjerland, G.Ø. 2006. Quantifying training intensity distribution in elite endurance athletes: is there evidence for an "optimal" distribution? Scand. J. Med. Sci. Sports. 16(1): 49-56. doi:10.1111/j.1600-0838.2004.00418.x. PMID:16430681.

Seiler, S., Haugen, O., and Kuffel, E. 2007. Autonomic recovery after exercise in trained athletes: intensity and duration effects. Med. Sci. Sports Exerc. 39(8): 1366-1373. doi:10.1249/mss.0b013e318060f17d. PMID:17762370.

Shibahara, N., Matsuda, H., Umeno, K., Shimada, Y., Itoh, T., and Terasawa, K. 1996. The responses of skin blood flow, mean arterial pressure and R-R interval induced by cold stimulation with cold wind and ice water. J. Auton. Nerv. Syst. 61(2): 109-115. doi:10.1016/S0165-1838(96)00065-3. PMID:8946327.

Stanley, J., Buchheit, M., and Peake, J.M. 2011. The effect of post-exercise hydrotherapy on subsequent exercise performance and heart rate variability. Eur. J. Appl. Physiol. 2(3): 951-961. doi:10.1007/s00421-011-2052-7. PMID: 21710292.

Stuckey, M.I., Tordi, N., Mourot, L., Gurr, L.J., Rakobowchuk, M., Millar, P.J., et al. 2012. Autonomic recovery following sprint interval exercise. Scan. J. Med. Sci. Sports, 22(6): 756-763. doi:10.1111/j.1600-0838.2011.01320.x. PMID:21535187.

Ste-Marie, D.M., and Lee, T.D. 1991. Prior processing effects on gymnastic judging. J. Exp. Psychol. Learn. Mem. Cogn. 17(1): 126-136. doi:10.1037/0278-7393. 17.1.126.

Toubekis, A.G., Tsolaki, A., Smilios, I., Douda, H.T., Kourtesis, T., and Tokmakidis, S.P. 2008. Swimming performance after passive and active recovery of various durations. Int. J. Sports Physiol. Perform. 3(3): 375-386. PMID:19211948.

Uusitalo, A.L., Uusitalo, A.J., and Rusko, H.K. 2000. Heart rate and blood pressure variability during heavy training and overtraining in the female athlete. Int. J.Sports. Med. 21(1): 45-53. doi:10.1055/s-2000-8853. PMID:10683099.

Westerlund, T., Uusitalo, A., Smolander, J., and Mikkelsson, M. 2006. Heart rate variability in women exposed to very cold air $\left(-110^{\circ} \mathrm{C}\right)$ during whole-body cryotherapy. J. Therm. Biol. 31(4): 342-346. doi:10.1016/j.jtherbio.2006.01.004.

Yamamura, C., Zushi, S., Takata, K., Ishiko, T, Matsui, N., and Kitagawa, K. 1999. Physiological characteristics of well-trained synchronized swimmers in relation to performance scores. Int. J. Sports Med. 20(4): 246-251. doi:10.1055/s2007-971125. PMID:10376481.

Yamamura, C., Matsui, N., and Kitagawa, K., 2000. Physiological loads in the team technical and free routines of synchronized swimmers. Med. Sci. Sports Exerc. 32(6): 1171-1174. doi:10.1097/00005768-200006000-00022. PMID:10862548. 